\title{
Absolute Strain at the Nanoscale from HREM Images
}

\author{
C.L. Johnson, ${ }^{*}$ E. Snoeck, ${ }^{* *}$ and M.J. Hÿtch** \\ * Centralized Research Facilities, Drexel University, Philadelphia, PA 19104 \\ ** CEMES-CNRS, 29, rue Jeanne Marvig, 31055 Toulouse, France
}

Geometric phase analysis (GPA) of high-resolution electron microscopy (HREM) images is one of the most commonly employed techniques for determining nanoscale strain distributions in a variety of materials. GPA maps the displacement of lattice fringes in an image relative to a reference lattice [1]. In practice, the reference lattice is commonly determined from a region in the image, such as a substrate, that is assumed to be strain free. However, in many cases, materials do not contain strainfree regions that are suitable for use as a reference. Interfaces and dislocations can have strain fields that extend far in to the surrounding materials. Commonly, nanocrystals and nanostructured materials strained throughout the sample volume. An example is five-fold-twinned decahedral Au nanoparticles [2].

Decahedral nanoparticles of f.c.c. materials commonly exhibit five-fold twinning about the [110] axis. In the simple solid-geometric model, the decahedra are composed of five tetrahedral subunits with $\{111\}$ crystallographic faces. In addition to sharing one edge coinciding with the five-fold [110] axis, the subunits are joined to adjacent tetrahedral through twin boundaries parallel to $\{111\}$ planes. However, tetrahedra packed in this manor do not completely fill space and a solid angle deficiency remains. The solid-angle deficiency is accommodated by a disclination, which results in an inhomogenous strain distributed throughout the particle. We have reported the relative rotation and shear strains in a decahedral Au nanoparticle (Fig. 1) measured by GPA and aberrationcorrected HREM [2]. However, the radial and tangential strain distributions determined with respect to an internal reference were not uniquely determined.

Recently, we have developed a method for determining the absolute radial and tangential strains (Fig. 2) using an external reference. We will present our method and outline the experimental challenges that must be met to obtain absolute strain distributions. Furthermore, we will show examples, such as the Au nanoparticle, for which the absolute strain is necessary to uniquely determine the material structure. The ability to determine absolute strains is critical for many materials research problems.

\section{References}

[1] GPA phase plug-in for DigitalMicrograph (Gatan) available from HREM Research: www.hremresearch.com

[2] C.L. Johnson et al, Nature Mater. 7 (2008) 120.

[3] This research was supported by the EU Integrated Infrastructure Initiative ESTEEM (Ref. 026019 ESTEEM). 


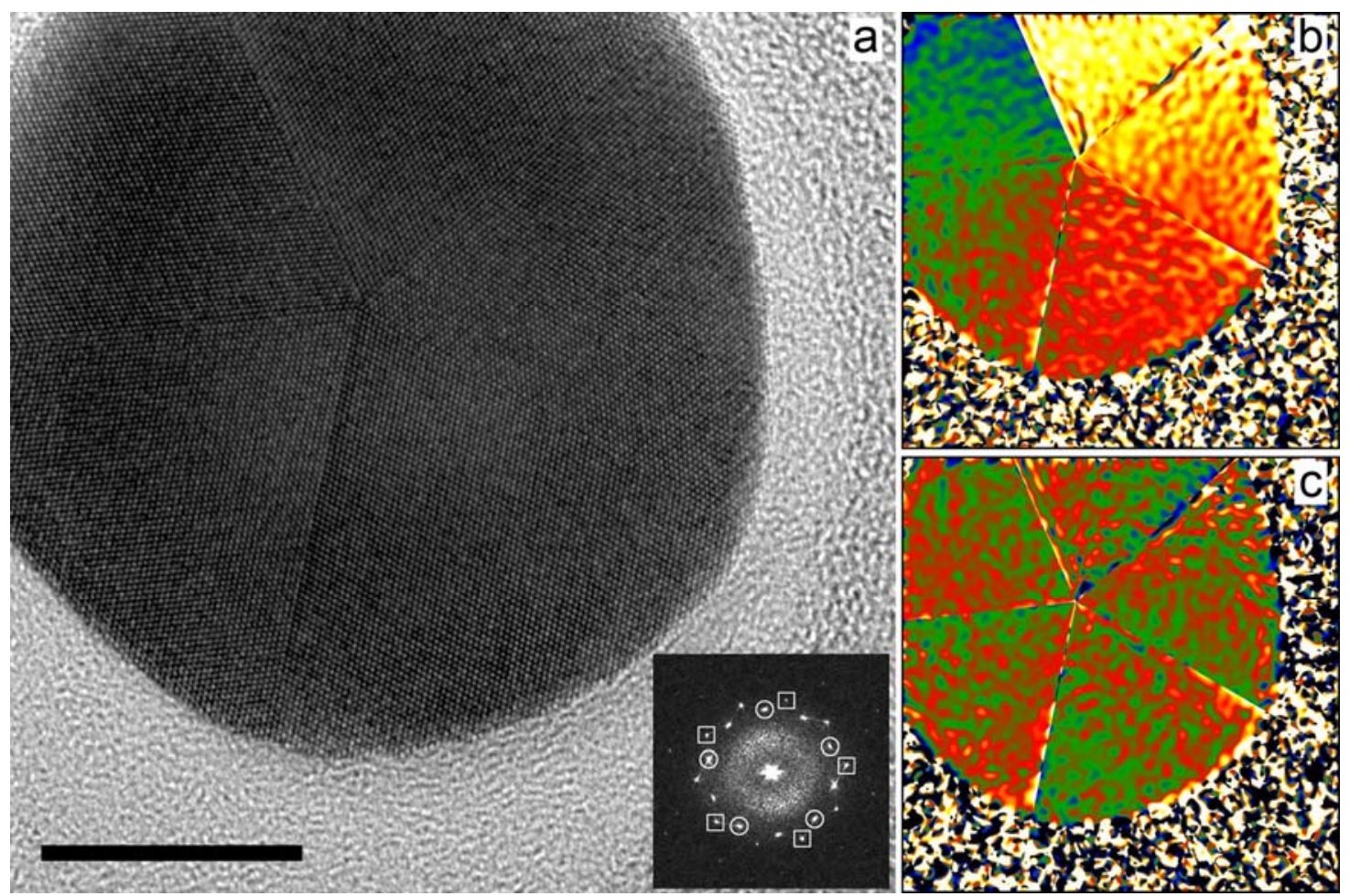

FIG. 1. Relative lattice rotation and strain in a decahedral gold nanoparticle. (a) The aberrationcorrected HREM image of the Au nanoparticle was used to determine the relative radial and tangential strains. Scale bar is $10 \mathrm{~nm}$. (b) The rotation map gives the internal rigid-body rotation of the crystallographic lattice and confirms the presence of a disclination. (c) The shear-strain map shows the shear gradient across each segment of the decahedral particle.

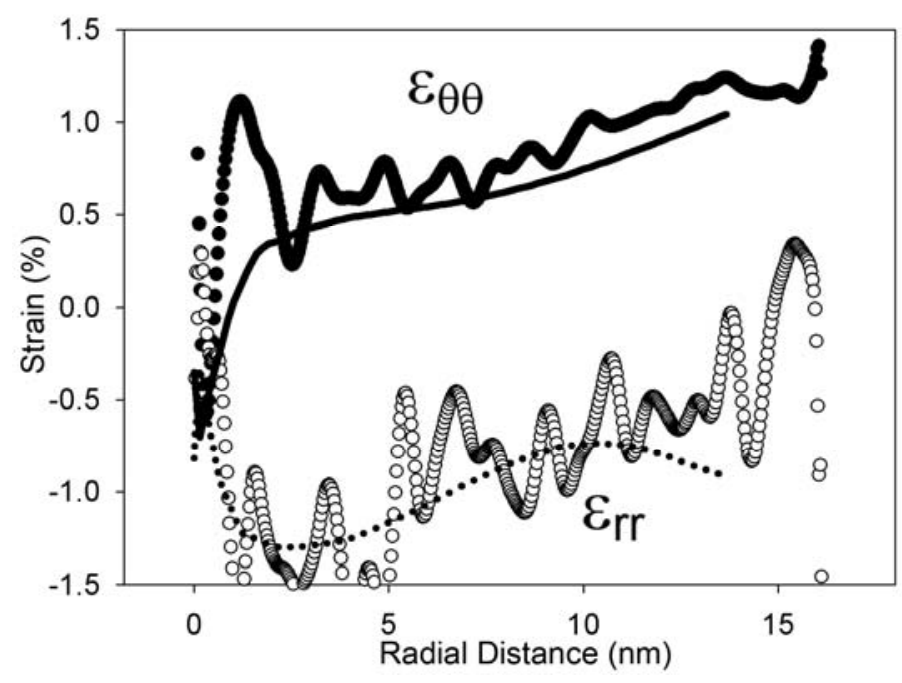

FIG. 2. Profiles of absolute radial $\left(\varepsilon_{\mathrm{rr}}\right)$ and tangential $\left(\varepsilon_{\theta \theta}\right)$ strains from the center of the particle to the edge (average of all the tetrahedral segments in Fig. 1) compared with those predicted by finite element modeling. The absolute strains reveal that the structure is compressed in the radial direction and stretched in the tangential direction throughout its volume. The difference between the radial and tangential strain is consistent with the finite element predictions. 